\title{
Regulatory Sub-Domain
}

National Cancer Institute

\section{Source}

National Cancer Institute. Regulatory Sub-Domain. NCI Thesaurus. Code C93385.

The Regulatory sub-domain is intended for those involved in the creation and review of submissions to regulatory authorities. The majority of business requirements come from the regulated product submission (RPS) message specification. It focuses on the documentation required for a product submission to the Food and Drug Administration (FDA). 